Pacific Journal of Mathematics

ON COMPLETENESS AND SEMICOMPLETENESS OF FIRST 


\title{
ON COMPLETENESS AND SEMICOMPLETENESS OF FIRST COUNTABLE SPACES
}

\author{
JOYLYN REED
}

\begin{abstract}
In this paper, well known completeness conditions in Moore spaces are generalized to arbitrary first countable spaces. Relationships are established between these conditions and various other completeness concepts including Cech completeness, countable completeness, and countable subcompactness. Finally, conditions are given for embedding a given first countable space in a "first countable complete" space. As one application of the theory developed, a necessary and sufficient condition is obtained for the embedding of a Moore space in a semicomplete or "Rudin" complete Moore space.
\end{abstract}

There has been considerable work done concerning complete Moore spaces, i.e., spaces satisfying R. L. Moore's Axiom 1, and completable Moore spaces, i.e., spaces which are dense subspaces of complete Moore spaces. There has also been much interest in M. E. Estill Rudin's concept of semicomplete Moore spaces, i.e., spaces satisfying Axiom $1^{\prime \prime}$. In this paper the author applies the concepts of completeness and semicompleteness to more general first countable spaces and establishes some theorems involving these concepts. Embedding theorems are also given. The last theorem of the paper answers a question discussed by Steve Armentrout at the Arizona State Topology Conference in 1967 by supplying a necessary and sufficient condition for a Moore space to be a dense subspace of a semicomplete Moore space.

The lower case letters $m, n, i, j$, and $k$ will denote positive integers unless otherwise stated.

Definition 1. The statement that the sequence $G_{1}, G_{2}, G_{3}, \cdots$ is an f.c. development for the space $X$ means that for each $n, G_{n}=$ $\left\{g_{i}(x) \mid i \geqq n, x \in X\right\}$, where for each $x \in X, g_{1}(x), g_{2}(x), g_{3}(x), \cdots$ is a sequence of open sets forming a local base at $x$.

Definition 2. The f.c. development is complete (semicomplete) provided that if $M_{1}, M_{2}, M_{3}, \cdots$ is a sequence of sets such that for each $n, M_{n}$ is a closed set contained in some element $g_{n}$ of $G_{n}$ and contains $M_{n+1}\left(M_{n}\right.$ is an element of $G_{n}$ and contains $\left.\overline{M_{n+1}}\right)$ then $\bigcap_{i=1}^{\infty} M_{i} \neq \varnothing$. The first countable space is complete (semicomplete) if and only if it has an f.c. complete (semicomplete) development.

THEOREM 1. A $T_{2}$ regular first countable semicomplete space $X$ 
satisfies Baire's theorem (the intersection of countably many dense open sets is a dense subset of the space).

Proof. For each $x \in X$, let $g_{1}(x), g_{2}(x), \cdots$ denote a local base for $x$ such that the corresponding f.c. development $G_{1}, G_{2}, \cdots$ is semicomplete. Let $D=d_{1}, d_{2}, \cdots$ denote a countable collection of open sets, each dense in $X$, and let $R$ be an open set. $R$ contains a point $p_{1}$ of $d_{1}$. Let $g_{n_{1}}\left(p_{1}\right) \in G_{1}$ such that $p_{1} \in g_{n_{1}}\left(p_{1}\right)$ and $\overline{g_{n_{1}}\left(p_{1}\right)} \cong R \cap d_{1}$. For $i>1$, let $p_{i} \in g_{n_{i-1}}\left(p_{i-1}\right) \cap d_{i}$ and let $g_{n_{i}}\left(p_{i}\right) \in G_{i}$ such that $p_{i} \in g_{n_{i}}\left(p_{i}\right)$ and $\overline{g_{n_{i}}\left(p_{i}\right)} \cong g_{n_{i-1}}\left(p_{i-1}\right) \cap d_{i}$. Notice that for each $i, g_{n_{i}}\left(p_{i}\right) \cong d_{i} \cap R$ and $\overline{g_{n_{i}}\left(p_{i}\right)} \subseteq g_{n_{i-1}}\left(p_{i-1}\right)$. Thus $\bigcap_{i=1}^{\infty} g_{n_{i}}\left(p_{i}\right) \neq \varnothing$. Since $\bigcap_{i=1}^{\infty} g_{n_{i}}\left(p_{i}\right) \subseteq$ $\left(\bigcap_{i=1}^{\infty} d_{i}\right) \cap R$, the intersection of $D$ is a dense subset of the space.

The reader should compare the following two theorems with those analogous theorems of Creede in [4].

THEOREM 2. A completely regular f.c. complete space $X$ with a $G_{\delta}$ diagonal is $\check{C}$ ech complete.

Proof. Let $Y$ be a $T_{2}$ compact space such that $X$ is a dense subspace of $Y$. For each $x \in X$, let $g_{1}(x), g_{2}(x), \cdots$ be a sequence of open sets of $X$ forming a local base at $x$ such that the corresponding f.c. development is complete, and $\{x, y\} \subseteq \bigcap_{i=1}^{\infty} g_{i}\left(x_{i}\right)$ implies $x=y$. For each $x \in X$ and positive integer $i$, let $G_{i}(x)$ be an open set of $Y$ such that $G_{i}(x) \cap X=g_{i}(x)$, and let $H_{i}(x)$ be an open set of $Y$ containing $x$ such that $\overline{H_{i}(x)} \subseteq G_{i}(x)$ (in $Y$ ). Notice $\overline{H_{i}(x) \cap X} \cap X \leqq G_{i}(x) \cap X$. For each positive integer $i$, let $H_{i}=\bigcup\left\{H_{i}(x) \mid x \in X\right\}$. Thus $H_{1}, H_{2}$, $H_{3}, \cdots$ is a sequence of open sets in $Y$ such that $X \subseteq \bigcap_{i=1}^{\infty} H_{i}$.

Assume $p \in \bigcap_{i=1}^{\infty} H_{i}$. For each $i$, let $H_{i}\left(x_{i}\right)$ contain $p$. Let $A_{i}=$ $\overline{H_{i}\left(x_{i}\right) \cap X} \cap X$. Since $X$ is dense in $Y, A_{1}, A_{2}, A_{3}, \cdots$ is a sequence of nonempty closed sets of $X$ with the finite intersection property such that for each $i, A_{i} \leqq g_{i}\left(x_{i}\right)$. Thus there is a point $x$ of $X$ such that $x \in \bigcap_{i=1}^{\infty} A_{i}$. Assume $x \neq p$. For each $i$, let $R_{i}$ be an open set of $Y$ containing $p$ such that $\overline{R_{i}}$ (in $Y$ ) does not contain $x$ and $\overline{R_{i}} \subseteq H_{i}\left(x_{i}\right)$. Let $B_{i}=\overline{R_{i} \cap X} \cap X$. As before, $\bigcap_{i=1}^{\infty} B_{i}$ contains some point $k$ of $X$. But $\{x, k\} \subseteq \bigcap_{i=1}^{\infty} g_{i}\left(x_{i}\right)$, so $x=k$. However, $x \neq k$ since $x \notin \overline{R_{i}}$ and $k \in B_{i} \subseteq \overline{R_{i}}$. Thus it must be that $x=p$ and $p \in X$. Hence $\bigcap_{i=1}^{\infty} H_{i}=X$.

THEOREM 3. A Čech complete first countable space $X$ is f.c. complete.

Proof. Let $Y$ be a $T_{2}$ compact space such that $X$ is a $G_{\delta}$ set in $Y$. Let $P_{1}, P_{2}, \cdots$ be a sequence of open sets of $Y$ such that $X=\bigcap_{i=1}^{\infty} P_{i}$. For each $x \in X$, let $g_{1}(x), g_{2}(x), \cdots$ be a sequence of 
open sets of $X$ forming a local base at $x$, such that for each $i$, $\overline{g_{i+1}(x)} \leqq g_{i}(x)$. For each $x \in X$ and positive integer $i$, let $G_{i}(x)$ be an open set in $Y$ such that $g_{i}(x)=G_{i}(x) \cap X$, and let $H_{i}(x)$ be an open set of $Y$ containing $x$ such that $\overline{H_{i}(x)} \cong G_{i}(x) \cap P_{i}$ and $\overline{H_{i+1}(x)} \leqq H_{i}(x)$. Thus $h_{1}(x), h_{2}(x), \cdots$ is a local base at $x$ in $X$ where for each $i, h_{i}(x)=$ $H_{i}(x) \cap X$. For each positive integer $i$, let $H_{i}=\left\{h_{n}(x) \mid n \geqq i, x \in X\right\}$. Thus $H_{1}, H_{2}, H_{3}, \cdots$ is an f.c. development for $X$.

Let $A_{1}, A_{2}, A_{3}, \cdots$ be a monotonically decreasing sequence of closed sets of $X$ such that for each $i, A_{i} \subseteq h_{n_{i}}\left(x_{i}\right) \in H_{i}$. The sequence $C l Y A_{1}$, $C l Y A_{2}, C l Y A_{3}, \cdots$, where $C l Y A_{i}$ denotes the closure in $Y$ of $A_{i}$, is a sequence of closed sets in $Y$ with the finite intersection property; hence, there is a point $y$ of $Y$ such that $y \in \bigcap_{i=1}^{\infty} C l Y A_{i}$. For each $i, C l Y A_{i} \leqq C l Y\left(H_{n_{i}}\left(x_{i}\right) \cap X\right) \leqq P_{i}$. Thus $y \in \bigcap_{i=1}^{\infty} P_{i}$ and hence $y \in X$. Thus $H_{1}, H_{2}, H_{3}, \cdots$ is a complete development for $X$.

In first countable spaces, the concepts of f.c. completeness and f.c. semicompleteness are related to Frolic's concept of countable completeness [6] and de Groot's concept of countable subcompactness [7]. To avoid confusion the term "countable Čech-completeness", coined by Lutzer and Aarts [1] will be used for the term "countable completeness".

The phrase " $C$ is a centered system on $X$ " means " $C$ is a collection of subsets of $X$ such that for any finite $C_{0} \leqq C, \cap C_{0} \neq \varnothing$ ". A collection $F$ of nonempty subsets of $X$ is called a regular filterbase [7] if whenever $F_{1}, F_{2} \in F$, some $F_{3} \in F$ has $\bar{F}_{3} \subseteq F_{1} \cap F_{2}$. A regular space $X$ is countably Čech-complete [6] if there is a sequence $\left\{\hat{B}_{n}\right\}$ of open bases for $X$ such that if $n_{1}<n_{2}<n_{3}<\cdots$ and if the sequence $\left\{B_{n_{k}}\right\}$, where $B_{n_{k}} \in \hat{B}_{n_{k}}$ forms a centered system, then $\bigcap\left\{\bar{B}_{n_{k}}\right\}=\varnothing$. A regular space is countably subcompact [7] with respect to a base $B$ of open sets provided that any countable regular filterbase $F \subseteq B$ has $\cap F \neq \varnothing$.

THEOREM 4. In regular first countable spaces, the following implications hold:

(1) f.c. completeness $\Longrightarrow$ (2) countable Čech-completeness (3) f.c. semicompleteness .

None of the above implications are reversible. In Moore spaces, countable Čech-completeness $\Leftrightarrow$ More completeness $\Leftrightarrow f . c$. completeness [1], and Rudin completeness $\Leftrightarrow$ f.c. semicompleteness. M. E. Rudin's example [5] of a Rudin complete space that is not Moore complete shows $(3) \nRightarrow(2)$. The following example shows $(2) \nRightarrow(1)$.

Example A. The Sorgenfrey line is the topological space $S$ of 
real numbers topologized by taking sets of the form $[a, b)$ to be basic open sets. Let $D_{1}, D_{2}, D_{3}, \cdots$ be a monotonic sequence of open sets on the line such that $\bigcap_{i=1}^{\infty} D_{i}=I$, where $I$ is the set of irrationals. Let $r_{1}, r_{2}, r_{3}, \cdots$ denote the rationals. For each $x \in I$, let $\left\{\left[x, x_{i}\right)\right\}$ be a sequence of open sets closing on $x$ such that for each $i, x_{\imath}$ is a rational not in $\left\{r_{1}, r_{2}, \cdots, r_{i}\right\}$. For each rational $x$, let $\left\{\left[x, x_{2}\right)\right\}$ be a sequence of open sets closing on $x$ such that for each $i, x_{i}$ is a rational, and $\left[x, x_{2}\right) \cap\left(\left\{r_{1}, r_{2}, \cdots, r_{2}\right\}-\{x\}\right)=\varnothing$.

For each positive integer $n$, let $B_{n}=\left\{\left[x, x_{2}\right) / x \in S, i \geqq n\right\}$. Notice that if $r$ is a rational there exists an $n$ such that for $i \geqq n, r \in g \in B_{i}$ implies $g=\left[r, x_{i}\right)$ for some $x_{i}$. Let $\left\{\left[p_{i}, q_{2}\right)\right\}$ be a centered sequence such that for each $i,\left[p_{2}, q_{i}\right) \in B_{i}$. Let $x \in \bigcap\left\{\left[p_{i}, q_{2}\right]\right\}$. Due to the construction of $\left\{B_{n}\right\}, x \in \bigcap\left\{\left[p_{i}, q_{i}\right)\right\}$. Thus $S$ is a first countable, countably Čech-complete space.

Now let $G_{1}, G_{2}, G_{3}, \cdots$ represent any f.c. development for $S$. There must exist a monotonically decreasing sequence having the following properties. (1) the $n$th term of $S$ belongs to $G_{n}$ and contains its left endpoint, $p_{n}$, and (2) $p_{1}, p_{2}, p_{3}, \cdots$ is an increasing sequence converging to a number $x$ on the line. The sequence $\left\{\left[p_{i}, x\right)\right\}$ is a monotonically decreasing sequence of closed sets such that the $n$th term is a subset of some $g \in G_{n}$, but has no common part. Thus $S$ is not f.c. complete.

The following theorems follow readily.

TheOREm 5. Any open subspace of an f.c. complete (f.c. semicomplete) is f.c. complete (f.c. semicomplete).

THEOREM 6. A $G_{o}$ subspace of a regular f.c. complete space is f.c. complete.

In Moore spaces, Rudin completeness $\Leftrightarrow$ countable subcompactness. In regular first countable spaces, countable subcompactness implies f.c. semicompleteness. In [1], Lutzer and Aarts use the term "completeness property" for any property implying the Baire property. Of the several completeness properties they examine, all but countable subcompactness and subcompactness are such that a space may have the property locally but fail to have it globally. The proof they give showing that if a regular space is locally countably subcompact then it is countably subcompact may be slightly altered to yield this theorem.

THEOREM 7. If a regular first countable space is locally f.c. complete, then it is f.c. semicomplete.

It would be interesting to know if in first countable spaces, f.c. 
semicompleteness implies countable subcompactness.

Some technical definitions are needed for the following theorems.

Definitions. (a) If $G=G_{1}, G_{2}, G_{3}, \cdots$ is a sequence of open sets, the sequence $g_{1}, g_{2}, \cdots$ is a nested sequence ( $g$-sequence) wrt $G$ if and only if for each $i, g_{i} \in G_{i}$ and contains $\overline{g_{i+1}}\left(g_{i} \in G_{i}\right.$ and contains $\left.g_{i+1}\right)$. (b) The set sequence $g_{1}, g_{2}, \cdots$ is adjacent to the set $M$ if and only if for each $i, g_{i} \cap M \neq \varnothing$. (c) The set sequences $g_{1}, g_{2}, g_{3}, \cdots$ and $k_{1}, k_{2}, k_{3}, \cdots$ are mutually separated if and only if for some $i, g_{\imath} \cap$ $k_{i}=\varnothing$. (d) The open set $D$ covers the set sequence $g_{1}, g_{2}, g_{3}, \cdots$ if and only if for some $i, g_{i} \subseteq D$.

THEOREM 8. Each $T_{2}$ first countable space is a dense subspace of an f.c. complete space.

Proof. Let $X$ be a first countable space. For each $x \in X$, let $g_{1}(x), g_{2}(x), \cdots$ be a monotonically decreasing sequence of open sets forming a local base at $x$, and let $G=G_{1}, G_{2}, G_{3}, \cdots$ be the corresponding f.c. development. The statement that $g_{1}, g_{2}, \cdots$ is an $f$-sequence means for each $n, g_{n}=\bigcap_{i=1}^{n} g_{i}^{\prime}$ for some sequence $g_{1}^{\prime}, g_{2}^{\prime}, g_{3}^{\prime}$, ... where for each $i, g_{i}^{\prime} \in G_{i}$. If each of $g=g_{1}, g_{2}, g_{3}, \cdots$ and $k=$ $k_{1}, k_{2}, k_{3}, \cdots$ is an $f$-sequence then we will say $g \sim k$ if and only if for each positive integer $n$ there exist positive integers $i$ and $j$ such that $k_{i} \subseteq g_{n}$ and $g_{j} \subseteq k_{n}$. If $g$ is an $f$-sequence, let $g^{\prime}$ denote $\{k \mid g \sim k\}$. Let $X^{\prime}=\left\{g^{\prime} \mid g\right.$ is an $f$-sequence such that each term of $g$ is nonempty $\}$. For each open set $D$, let $D^{\prime}=\left\{g^{\prime} \mid D\right.$ covers $\left.g\right\}$. Let $0=\left\{D^{\prime} \mid D\right.$ is open in $X\}$ be a basis for a topology $\Omega$ on $X$.

The ordered pair $\left(X^{\prime}, \Omega\right)$ is a topological space. $X$ is a dense subspace of $X^{\prime}$ because $X$ is homeomorphic to the subspace $\left\{I(x)^{\prime} \mid x \in\right.$ $\left.X, I(x)=g_{1}(x), g_{2}(x), \cdots\right\} . \quad X^{\prime}$ is first countable. For each $I^{\prime} \in X^{\prime}$, where $I=g_{1}, g_{2}, \cdots$, and for each positive integer $n$, let $D_{n}\left(I^{\prime}\right)=g_{n}$. Thus $D_{1}\left(I^{\prime}\right)^{\prime}, D_{2}\left(I^{\prime}\right)^{\prime}, D_{3}\left(I^{\prime}\right)^{\prime}, \cdots$ forms a local base at $I^{\prime}$ in $X^{\prime}$.

$X^{\prime}$ is f.c. complete. For each positive integer $i$, let $G_{i}^{\prime}=$ $\left\{D_{n}\left(I^{\prime}\right)^{\prime} \mid n \geqq i, I^{\prime} \in X^{\prime}\right\}$. Let $M_{1}, M_{2}, M_{3}, \cdots$ be a monotonically decreasing sequence of closed sets of $X^{\prime}$ such that for each $i, M_{i} \leqq$ $D_{n_{i}}\left(y_{i}^{\prime}\right)^{\prime}$ for some $D_{n_{i}}\left(y_{i}^{\prime}\right)^{\prime} \in G_{i}^{\prime}$. Since $\bigcap_{i=1}^{m} D_{n_{i}}\left(y_{i}^{\prime}\right)^{\prime} \neq \varnothing$ for each $m$, then $\bigcap_{i=1}^{m} D_{n_{i}}\left(y_{i}^{\prime}\right) \neq \varnothing$. Now for each $i, D_{n_{i}}\left(y_{i}^{\prime}\right)=\bigcap_{k=1}^{n_{i}} g_{k}\left(y_{i}\right)$ for some sequence $g_{1}\left(y_{i}\right), g_{2}\left(y_{i}\right), g_{3}\left(y_{i}\right), \cdots$ where $g_{k}\left(y_{i}\right) \in G_{k}$. Thus $D_{n_{i}}\left(y_{i}^{\prime}\right) \leqq g_{i}\left(y_{i}\right)$ since $n_{i} \geqq i$. Since $\bigcap_{i=1}^{m} D_{n_{i}}\left(y_{2}\right) \neq \varnothing$ for each $m$, then $\bigcap_{i=1}^{m} g_{i}\left(y_{i}\right) \neq \varnothing$. Observe that $g_{i}\left(y_{i}\right) \in G_{i}$, so $\bigcap_{i=1}^{1} g_{i}\left(y_{i}\right), \bigcap_{i=1}^{2} g_{i}\left(y_{i}\right), \bigcap_{i=1}^{3} g_{i}\left(y_{2}\right), \cdots$ is an $f$-sequence. Let $J$ denote this $f$-sequence and examine any open set $D^{\prime}$ of $X^{\prime}$ containing $J^{\prime} . \quad D$ contains $\bigcap_{i=1}^{k} g_{2}\left(y_{i}\right)$ for some $k$. So $D$ contains $\bigcap_{i=1}^{k} D_{n_{i}}\left(y_{i}^{\prime}\right)$, and thus $D^{\prime}$ contains $\bigcap_{i=1}^{k} D_{n_{i}}\left(y_{i}^{\prime}\right)^{\prime}$ and $M_{k}$. $J^{\prime}$ is thus a point or limit point of $M_{n}$ for each $n$, and hence $J^{\prime} \in \bigcap_{i=1}^{\infty} M_{i}$. 
Thus, $G_{1}^{\prime}, G_{2}^{\prime}, G_{3}^{\prime}, \cdots$ is an f.c. development for $X^{\prime}$.

TheOREM 9. Each regular $T_{2}$ space $X$ is a dense subspace of a $T_{2}$ f.c. semicomplete space.

Proof. For each $x \in X$, let $I(x)=g_{1}(x), g_{2}(x), \cdots$ be a nested sequence of open sets forming a local base at $x$. Let $G=G_{1}, G_{2}, G_{3}$, .. be the corresponding f.c. development for $X$.

If $I$ is a nested sequence wrt $G$, let $I^{\prime}=\{K \mid K$ is a nested sequence wrt $G$ and $K \sim I\}$ (refer to the technical definitions and the proof of Theorem 8). Notice that $x \neq y$ implies $I(x)$ and $I(y)$ are mutually separated.

Let $K$ denote a maximal collection of mutually separated nested sequences wrt $G$ such that for each $x, I(x) \in K$. * Let $K^{\prime}=\left\{k^{\prime} \mid k \in K\right\}$. If $D$ is an open set, let $D^{\prime}=\left\{k^{\prime} \mid D\right.$ covers $k$ and $\left.k \in K\right\}$. Let $B=$ $\left\{D^{\prime} \mid D\right.$ is open in $\left.X\right\}$ be a basis for a topology $\Omega$ on $K^{\prime} . \quad X$ is a dense subspace of the $T_{2}$ space $\left(K^{\prime}, \Omega\right)$.

$K^{\prime}$ is first countable. For each $I^{\prime} \in K^{\prime}$, where $I=g_{1}, g_{2}, g_{3}, \cdots$, and each positive integer $n$, let $D_{n}\left(I^{\prime}\right)=g_{n}$. Thus $D_{1}\left({ }^{\prime}\right)^{\prime}, D_{2}\left(I^{\prime}\right)^{\prime}, \cdots$ forms a local base at $I^{\prime}$ in $K^{\prime}$.

$K^{\prime}$ is f.c. semicomplete. Examine the f.c. development for $K^{\prime}$, $G^{\prime}=G_{1}^{\prime}, G_{2}^{\prime}, \cdots$, where for each $i, G_{i}^{\prime}=\left\{D_{n}\left(I^{\prime}\right)^{\prime} \mid n \geqq i, I^{\prime} \in K^{\prime}\right\}$. Let $M=D_{n_{1}}\left(I_{1}^{\prime}\right)^{\prime}, D_{n_{2}}\left(I_{2}^{\prime}\right)^{\prime}, \cdots$ be a nested sequence wrt $G^{\prime}$, i.e., for each $i$, $\overline{D_{n_{i+1}}\left(I_{i+1}^{\prime}\right)^{\prime}} \leqq C_{n_{i}}\left(I_{i}^{\prime}\right)^{\prime}$ and $D_{n_{i}}\left(I_{i}^{\prime}\right)^{\prime} \in G_{i}^{\prime}$. Now for each $i, D_{n_{i}}\left(I_{i}^{\prime}\right)^{\prime}=g_{m_{i}}\left(x_{i}\right)^{\prime}$ for some $g_{m_{i}}\left(x_{i}\right) \in G_{i}$. Since $\overline{g_{m_{i+1}}\left(x_{i+1}\right)^{\prime}} \leqq g_{m_{i}}\left(x_{i}\right)^{\prime}$ in $K^{\prime}$, then $\overline{g_{m_{i}{ }_{1}}\left(x_{i+1}\right)} \subseteq$ $g_{m_{i}}\left(x_{2}\right)$ in $X$. Hence, $g_{m_{1}}\left(x_{1}\right), g_{m_{2}}\left(x_{2}\right), g_{m_{3}}\left(x_{3}\right), \cdots$ is a nested sequence wrt $G$ in $X$. Since $K$ is maximal, there is an element $I=g_{1}, g_{2}, g_{3}$, .. of $K$ such that for each $k, g_{m_{k}}\left(x_{k}\right) \cap g_{k} \neq \varnothing$. Let $D^{\prime}$ be an open set of $K^{\prime}$ containing $I^{\prime} . \quad D$ contains $g_{i}$ for some $i$, and in fact $D$ contains $g_{k}$ for $k \geqq i$. Thus for all $k, D \cap g_{m_{k}}\left(x_{k}\right) \neq \varnothing$ and $D^{\prime} \cap g_{m_{k}}\left(x_{k}^{\prime}\right)^{\prime}=$ $D^{\prime} \cap D_{n_{k}}\left(I^{\prime}\right)^{\prime} \neq \varnothing$. So $I^{\prime} \in \overline{D_{n_{k}}\left(I_{k}^{\prime}\right)^{\prime}}$ for each positive integer $k$. Thus $I^{\prime} \in \bigcap_{i=1}^{\infty} D_{n_{i}}\left(I_{i}^{\prime}\right)^{\prime}$. Hence, $G^{\prime}$ is an f.c. semicomplete development for $K^{\prime}$.

Definition 3. Property $R$. A $T_{2}$ regular first countable space has property $R$ provided that there exists an f.c. development $G=$ $G_{1}, G_{2}, G_{3}, \cdots$ and a collection $K$ of mutually separated nested sequences wrt $G$ such that: (1) for each $x \in X$, some element of $K$ forms a local base at $x$, (2) if $g$ is a nested sequence wrt $G$, there is an element $k$ of $K$ such that $g$ and $k$ are not mutually separated, and (3) if the open set $D$ covers the sequence $g_{1}, g_{2}, g_{3}, \cdots$ of $K$, there is an integer $n$ such that $D$ covers any element $k$ of $K$ adjacent to $g_{n}$.

THEOREm 10. A $T_{2}$ regular first countable space $X$ with property 
$R$ is a dense subspace of a $T_{2}$ regular f.c. semicomplete space.

Proof. Let $G$ and $K$ respectively be the f.c. development and collection as assured by property $R$. That $X$ is a dense subspace of a $T_{2}$ f.c. semicomplete space can be seen by applying the part of the proof of Theorem 9 following *, using $K$ as defined here.

We will now see that the space $K^{\prime}$ is regular. Let $D^{\prime}$ be an open set containing $I^{\prime}$ of $K^{\prime}$, where $I=g_{1}, g_{2}, g_{3}, \cdots$. Thus $D$ covers $I$, and there is an integer $n$ such that if $k=k_{1}, k_{2}, \cdots$ of $K$ is adjacent to $g_{n}$, then $D$ covers $k$. Let $k^{\prime}$ be a limit point of $D_{n}\left(I^{\prime}\right)^{\prime}=g_{n}^{\prime}$. Thus $D_{1}\left(k^{\prime}\right)^{\prime}, D_{2}\left(k^{\prime}\right)^{\prime}, \ldots$ is adjacent to $D_{n}\left(I^{\prime}\right)^{\prime}$ and so $k_{1}, k_{2}, k_{3}, \cdots$ is adjacent to $g_{n}$. Thus $D$ covers $k$, and $k^{\prime} \in D^{\prime}$. So $\overline{D_{n}\left(I^{\prime}\right)^{\prime}} \subseteq D^{\prime}$.

THEOREM 11. Each $T_{2}$ regular f.c. semicomplete space $X$ has property $R$.

Proof. Let $G=G_{1}, G_{2}, \cdots$ be an f.c. semicomplete development for $X$. For each $x \in X$, let $I(x)=g_{1}(x), g_{2}(x), \cdots$ where for each $i$, $g_{i}(x) \in G_{i}$, and $I(x)$ forms a local base at $x$. Let $K=\{I(x) \mid x \in X\}$.

Let $g=g_{1}, g_{2}, \cdots$ be a nested sequence wrt $G$. Thus for some $x, x \in \bigcap_{i=1}^{\infty} g_{i}$. So $I(x) \in K$ and $g$ and $I(x)$ are not mutually separated.

Let $D$ be an open set covering $I(x)$ of $K$. Let $n$ be an integer such that $\overline{g_{n}(x)} \cong D$. Thus if $I(y)$ is adjacent to $g_{n}(x), y \in \overline{g_{n}(x)}$ and thus, $y \in D$. Thus $D$ covers $I(y)$. This completes the proof that $X$ satisfies property $R$.

Definition. If $X$ is a topological space, the statement that $G$ is a nested development for $X$ means that (1) for each positive integer $n, G_{n}$ is an open cover of $X$ containing $G_{n+1}$ as a subcover and (2) if $U$ is an open set and $p$ is any point of $U$ there is an integer $n$ such that $p \in g \in G_{n}$ implies $g \leqq U$.

Definition. The topological space $X$ is a Moore space if and only if it is a regular $T_{2}$ space with a nested development.

Definition. A complete (semicomplete) Moore space is a Moore space with a nested development $G$ having the property that if $M_{1}, M_{2}, M_{3}, \ldots$ is a sequence of sets such that for each $n, M_{n}$ is a closed set containing $M_{n+1}$ and $M_{n}$ is a subset of some element $g_{n}$ of $G_{n}\left(M_{n}\right.$ is an element of $G_{n}$ and contains $\left.\overline{M_{n+1}}\right)$, then $\bigcap_{i=1}^{\infty} M_{i} \neq \varnothing$. Such a development is called a complete (semicomplete) nested development.

Every complete Moore space is semicomplete, but the converse is not true [5]. Not every Moore space is a dense subspace of a 
complete Moore space [5]. In [9], Whipple provides a necessary and sufficient condition for a Moore space to be completable. Not every Moore space is a dense subspace of a semicomplete Moore space [5]. In his thesis for the University of Iowa, Alzoobaee provides a sufficient condition but it is not known if this condition is necessary for a Moore space to be semicompletable. The following definition and theorem provide a necessary and sufficient condition.

Definition 4. The Moore space $X$ satisfies Axiom $K$ provided that there exists a nested development $Q$ and a collection $K$ of mutually separated $g$-sequences wrt $Q$ such that: (1) for each $x \in X, x \in \cap g$ for some $g$ of $K$, (2) if $d$ is a nested sequence wrt $Q$, there is an element $g$ of $K$ such that $d$ and $g$ are not mutually separated, and (3) if the open set $D$ covers the sequence $d$ of $K$, there is an integer $n$ such that if $g_{1}, g_{2}, g_{3}, \cdots$ is an element of $K$ and $g_{n}$ covers $d$, then $D$ covers any element $k$ of $K$ adjacent to $g_{n}$. (The reader should refer to definitions $a, b$, and $c$ for explanation of above terms.)

Theorem 12. A Moore space $X$ satisfying Axiom $K$ is a dense subspace of a semicomplete Moore space.

Proof. Let $X$ be a Moore space with $Q=Q_{1}, Q_{2}, Q_{3}, \cdots$ and $K$ defined as in Definition 4. Form the topological space $\left(K^{\prime}, \Omega\right)$ as in the paragraph following * in the proof of Theorem 5, using $Q$ for $G$.

For each $k^{\prime} \in K^{\prime}$, where $k=k_{1}, k_{2}, k_{3}, \cdots$, let $g_{n}\left(k^{\prime}\right)=k_{n}$. As before, $g_{1}\left(k^{\prime}\right)^{\prime}, g_{2}\left(k^{\prime}\right)^{\prime}, \cdots$ forms a local base at $k^{\prime}$ in $K^{\prime}$. For each positive integer $n$, let $Q_{n}^{\prime}=\left\{g_{2}\left(k^{\prime}\right)^{\prime} \mid i \geqq n, k^{\prime} \in K^{\prime}\right\} . \quad Q^{\prime}=Q_{1}^{\prime}, Q_{2}^{\prime}, Q_{3}^{\prime}, \cdots$ is a nested development for $K^{\prime}$. Let $D^{\prime}$ be an open set containing $k^{\prime}$ of $K^{\prime}$. Thus $D$ covers $k_{1}, k_{2}, k_{3}, \cdots$ of $K$. There is an integer $n$ such that if $g_{1}, g_{2}, g_{3}, \cdots$ is an element of $K$ and $g_{n}$ covers $k_{1}, k_{2}, k_{3}$, $\cdots$, then $D$ covers any element $m_{1}, m_{2}, m_{3}, \cdots$ of $K$ adjacent to $g_{n}$. Let $d^{\prime}$ be an element of $Q_{n}^{\prime}$ containing $k^{\prime}$. Now $d=g_{t}\left(j^{\prime}\right)$ for some positive integer $t \geqq n$ and $j \in K$. Also $g_{t}\left(j^{\prime}\right)=j_{t}$ for some $j_{1}, j_{2}, j_{3}, \cdots$ $K$. Thus $j_{t}$ covers $k$. Assume $h^{\prime}$, where $h=h_{1}, h_{2}, h_{3}, \cdots$, is a point of limit point of $d^{\prime}$, i.e., of $j_{t}^{\prime}$. Thus $g_{1}\left(h^{\prime}\right)^{\prime}, g_{2}\left(h^{\prime}\right)^{\prime}, g_{3}\left(h^{\prime}\right)^{\prime}, \cdots$ is adjacent to $j_{t}^{\prime}$, and hence $h_{1}, h_{2}, h_{3}, \cdots$ is adjacent $j_{t}$. Thus $D$ must cover $h_{1}, h_{2}, h_{3}, \cdots$ and $h^{\prime} \in D^{\prime}$. This shows that $K^{\prime}$ is regular.

From techniques used in the proof of Theorem 5, we see that $K^{\prime}$ is $T_{2}$, contains $X$ as a dense subspace, and the development $Q$ is a semicomplete nested development for $K^{\prime}$. This completes the proof.

THEOREM 13. A dense subspace of a semicomplete Moore space $S$ satisfies Axiom $K$. 
Proof. In Theorem 2 of [5], M. E. Rudin proves the following result. If $S$ is a Moore space with nested development $G, S^{\prime}$ is a subspace of $S$ and $G^{\prime}$ is a nested development for $S^{\prime}$, there are sequences $T_{1}, T_{2}, T_{3}, \cdots$ and $Q_{1}, Q_{2}, Q_{3}, \cdots$ such that (1) $T_{i}$ and $Q_{i}$ are subcollections of $G_{i}$ and $G_{t}^{\prime}$ containing $T_{i+1}$ and $Q_{\imath+1}$ respectively covering $S^{\prime}$, (2) if $q_{1}, q_{2}, q_{3}, \cdots$ is a sequence such that for each $i$, $q_{i} \in Q_{i}$ and contains $q_{i+1}$, then there are sequences $w_{1}, w_{2}, \cdots$ of increasing integers and $g_{1}, g_{2}, g_{3}, \cdots$ of regions such that for each $i$, $g_{i} \in T_{i}$ and contains $\overline{g_{i+1}}$ and $\overline{q_{w_{i}}}$ (in $S$ ), and (3) if $x_{1}, x_{2}, x_{3}, \cdots$ is a sequence such that for each $i, x_{i} \in T_{i}$ and contains $x_{i+1}$, there are sequences $z_{1}, z_{2}, z_{3}, \cdots$ of increasing integers and $h_{1}, h_{2}, h_{3}, \cdots$ of regions such that for each $i, h_{i} \in Q_{i}$ and contains $\overline{h_{i+1}}$ and $\overline{x_{z_{i}}} \cap S^{\prime}$.

Let $S^{\prime}$ be a dense subspace of a semicomplete Moore space $S$. Let $M$ be a semicomplete nested development for $S$. Let $G_{1}=M_{1}$. For each positive integer $n \geqq 2$, let $G_{n}=\left\{g \mid g \in M_{n}\right.$ and $g$ is a subset of some element of $\left.G_{n-1}\right\}$. Thus $G=G_{1}, G_{2}, G_{3}, \cdots$ is also a semicomplete nested development for $S$. Let $G^{\prime}$ be the corresponding development for $S^{\prime}$. Applying the preceding theorem to $S, G, S^{\prime}$, and $G^{\prime}$, we get the sequences $T=T_{1}, T_{2}, T_{3}, \cdots$ and $Q=Q_{1}, Q_{2}, Q_{3}, \cdots$. Notice that $Q$ is a nested development for $S^{\prime}$.

For each element $x$ of $S$ such that $x \in \bigcap_{i=1}^{\infty} C l S q_{i}$ for some sequence $q_{1}, q_{2}, q_{3}, \cdots$ where $q_{i} \in Q_{i}$ and $q_{i+1} \subseteq q_{i}$, let $d_{x}$ denote one such sequence, $q_{1}, q_{2}, q_{3}, \cdots$. Let $w_{1}, w_{2}, w_{3}, \cdots$ and $g_{1}(x), g_{2}(x), \cdots$ be sequence such that $g_{i}(x) \in T_{2}$ and $g_{2}(x)$ contains $\overline{g_{i+1}(x)}$ and $\overline{q_{w_{2}}}$ in $S$. Notice $x \in \bigcap_{i=1}^{\infty} g_{i}(x)$. Now, let $z_{1}, z_{2}, z_{3}, \cdots$ and $h(x)=h_{1}(x), h_{2}(x), \cdots$ be sequences such that $h_{i}(x) \in Q_{i}$, contains $\overline{h_{i+1}(x)}$ and $\overline{g_{z_{i}}} \cap S^{\prime}$. For each $n, x$ is a point or limit point of $h_{n}(x)$ in $S$, since for each $k \geqq n$, $g_{z_{k}}(x)$ contains $x$ and intersects $h_{n}(x)$.

If $x \neq y$ and $h_{x}$ and $h_{y}$ exist, then $h(x)$ and $h(y)$ are mutually separated. Let $D_{x}$ and $D_{y}$ be mutually separated open sets in $S$ containing $x$ and $y$ respectively. Let $n$ be a positive integer such that any element of $G_{n}$ containing $x$ is a subset of $D_{x}$ and any element of $G_{n}$ containing $y$ is a subset of $D_{y}$. Let $m>n+1$. Now $x \in \overline{h_{m}(x)}$ and $h_{m}(x) \in G_{m}^{\prime} ; y \in \overline{h_{m}(y)}$ and $h_{m}(y) \in G_{m}^{\prime}$. In $S, \overline{h_{m}(x)}$ is a subset of some element of $G_{m-1}$ containing $x$ and $\overline{h_{m}(y)}$ is a subset of some element of $G_{m-1}$ containing $y$. Thus $h_{m}(x) \subseteq D_{x}$ and $h_{m}(y) \cong D_{y}$. So $h_{m}(x) \cap h_{m}(y)=\varnothing$.

Let $K=\{h(x) \mid x \in S, h(x)$ exists $\}$. Thus $K$ is a collection of mutually separated $g$-sequences wrt $Q$, where $Q$ is a nested development for $S^{\prime}$.

To examine $\# 3$ of Axiom $K$, let $D$ be an open set of $S^{\prime}$ covering $h(x)=h_{1}(x), h_{2}(x), h_{3}(x), \cdots$ of $K$. Let $m$ be the maximal open set in 
$S$ such that $m \cap S^{\prime}=D$. $D$ contains $h_{n}(x)$ for some $n$. Thus $D$ contains $\overline{g_{z_{n}}(x)} \cap S^{\prime}$, and $m$ contains $g_{z_{n}}(x)$. Thus $m$ contains $x$. Let $k$ be a positive integer such that $x \in g \in G_{k}$ implies $g \subseteq m$. Let $j>k+1$. Let $h_{1}(y), h_{2}(y), h_{3}(y), \cdots$ be an element of $K$ such that $h_{j}(y)$ covers $h(x)$. Since $x \in \overline{h_{i}(x)}$ in $S$, for each $i, x \in \overline{h_{i}(y)}$ in $S$. Since $\overline{h_{j}(y)}$ in $S$ is a subset of some element $g$ of $G_{j-1}$, then $x \in g$ and $g \leqq m$. Thus $\overline{h_{j}(y)} \subseteq m$ in $S$, and $y \in m$. Now let $h_{1}(p), h_{2}(p), h_{3}(p), \cdots$ be an element of $K$ adjacent to $h_{j}(y)$. The point $p$ is an element of $\overline{h_{\imath}(p)}$ in $S$ for each $i$. Let $m_{1}, m_{2}, m_{3}, \cdots$ be a sequence such that for each $i, m_{\imath} \in G_{2}$ and contains $\overline{h_{i+1}(p)}$ in $S$. Thus for each $i, m_{\imath} \cap h_{j}(y) \neq \varnothing$, since $h_{i+1}(p) \cap h_{j}(y) \neq \varnothing$. So $p \in \overline{h_{j}(y)}$ in $S$. Since $\overline{h_{j}(y)} \subseteq m, p \in m$. Thus for some $i, m$ contains $h_{\imath}(p)$. Hence $D$ contains $h_{\imath}(p)$ and covers $h(p)$.

To examine $¥ 2$ of Axiom $K$, let $q_{1}, q_{2}, q_{3}, \cdots$ be a nested sequence wrt $Q$ in $S^{\prime}$. Thus for each $n, q_{n} \in Q_{n}$ and contains $\overline{q_{n+1}}$. Let $w_{1}, w_{2}$, $w_{3}, \cdots$ and $g_{1}, g_{2}, g_{3}, \cdots$ be such that $g_{n} \in T_{n}$ and $g_{n} \supseteqq \overline{g_{n+1}}$ and $\overline{q_{w_{n}}}$ in $S$. Since $G$ is a semicomplete development for $S$, there is an $x$ such that $x \in \bigcap_{i=1}^{\infty} g_{i}$. Thus for each $i, x \in \bar{q}_{i}$ in $S$ since for $j \geqq i g_{j}$ contains $x$ and intersects $q_{i}$. Thus $x \in \bigcap_{i=1}^{\infty} \overline{q_{i}}$ in $S$, where $q_{i} \in Q_{i}$ and $q_{i}$ contains $q_{i+1}$. Examine $h(x)$. Let $q_{1}(x)^{\prime}, q_{2}(x)^{\prime}, q_{3}(x)^{\prime}, \cdots$ be the defining sequence for $h(x)$, i.e., $x \in \bigcap_{i=1}^{\infty} q_{i}(x)^{\prime}$ in $S$ and $q_{i}(x)^{\prime} \in Q_{\imath}$ and $q_{i+1}(x)^{\prime} \leqq q_{\imath}(x)^{\prime}$. The sequence $w_{1}, w_{2}, w_{3}, \cdots$ and $g_{1}(x), g_{2}(x), g_{3}(x), \cdots$ were chosen such that $g_{n}(x) \supseteq \overline{g_{n+1}(x)}$ and $\overline{q_{w_{n}}(x)^{\prime}}$ in $S$. Since for each $n, x \in g_{n}(x)$ and $\overline{x \in q_{n}(x)}$ in $S$, then $g_{n}(x) \cap q_{n}(x) \neq \varnothing$. Since $h_{n}(x)$ contains $\overline{g_{z_{n}}(x)} \cap S^{\prime}$, we have $h_{n}(x) \cap q_{z_{n}}(x) \neq \varnothing$, and hence, $h_{n}(x) \cap q_{n}(x) \neq \varnothing$ for each $n$. Thus there is an element $h(x)$ of $K$ such that $h(x)$ and $q_{1}, q_{2}, q_{3}, \cdots$ are not mutually separated.

This completes the proof that a Moore space is semicompletable if and only if it satisfies Axiom $K$.

\section{REFERENCES}

1. J. M. Arts and D. J. Lutzer, Completeness properties designed for recognizing Baire spaces, to appear.

2. O. H. Alzoobaee, Completions of Moore spaces, Thesis, University of Iowa, 1962.

3. S. Armentrout, Completing Moore spaces, Topology Conference, Arizona State University, 1967.

4. G. D. Creede, Embedding of complete Moore spaces, Proc. Amer. Math. Soc., 28 (1971), 609-612.

5. M. E. Estill (= M. E. Rudin), Concerning abstract spaces, Duke Math. J., 17 (1950), $317-327$.

6. Z. Frolik, Baire spaces and some generalizations of complete metric spaces, Czech Math. J., 11 (1961), 237-247.

7. J. De Groot, Subcompactness and the Baire category theorem, Indag. Math., 25 (1963), 761-767. 
8. R. L. Moore, Foundations of Point Set Theory, Amer. Math. Soc. Colloquium Publications, vol. 13, New York, 1932.

9. K. E. Whipple, Cauchy sequences in Moore spaces, Pacific J. Math., 18 (1966), 191-199.

Received March 30, 1973. This paper was written while the author was under the direction of Professor Phillip L. Zenor.

AUBURN UNIVERSITY 



\section{PACIFIC JOURNAL OF MATHEMATICS}

\section{EDITORS}

RICHARD ARENS (Managing Editor)

University of California

Los Angeles, California 90024

\section{R. A. Beaumont \\ University of Washington \\ Seattle, Washington 98105}

\section{J. DugundJI}

Department of Mathematics

University of Southern California

Los Angeles, California 90007

D. Gilbarg and J. Milgram

Stanford University

Stanford, California 94305

\section{ASSOCIATE EDITORS}
E. F. BECKENBACH
B. H. NeumanN
F. WOLF
K. YOSHIDA

\section{SUPPORTING INSTITUTIONS}

UNIVERSITY OF BRITISH COLUMBIA
CALIFORNIA INSTITUTE OF TECHNOLOGY
UNIVERSITY OF CALIFORNIA
MONTANA STATE UNIVERSITY
UNIVERSITY OF NEVADA
NEW MEXICO STATE UNIVERSITY
OREGON STATE UNIVERSITY
UNIVERSITY OF OREGON
OSAKA UNIVERSITY

UNIVERSITY OF BRITISH COLUMBIA

UNIVERSITY OF CALIFORNIA

MONTANA STATE UNIVERSITY

NEW MEXICO STATE UNIVERSITY

OREGON STATE UNIVERSITY

OSAKA UNIVERSITY

\author{
UNIVERSITY OF SOUTHERN CALIFORNIA \\ STANFORD UNIVERSITY \\ UNIVERSITY OF TOKYO \\ UNIVERSITY OF UTAH \\ WASHINGTON STATE UNIVERSITY \\ UNIVERSITY OF WASHINGTON \\ AMERICAN MATHEMATICAL SOCIETY \\ NAVAL WEAPONS CENTER
}

The Supporting Institutions listed above contribute to the cost of publication of this Journal, but they are not owners or publishers and have no responsibility for its content or policies.

Mathematical papers intended for publication in the Pacific Journal of Mathematics should be in typed form or offset-reproduced, (not dittoed), double spaced with large margins. Underline Greek letters in red, German in green, and script in blue. The first paragraph or two must be capable of being used separately as a synopsis of the entire paper. Items of the bibliography should not be cited there unless absolutely necessary, in which case they must be identified by author and Journal, rather than by item number. Manuscripts, in triplicate, may be sent to any one of the editors. Please classify according to the scheme of Math. Reviews, Index to Vol. 39. All other communications should be addressed to the managing editor, or Elaine Barth, University of California, Los Angeles, California, 90024.

The Pacific Journal of Mathematics expects the author's institution to pay page charges, and reserves the right to delay publication for nonpayment of charges in case of financial emergency.

100 reprints are provided free for each article, only if page charges have been substantially paid. Additional copies may be obtained at cost in multiples of 50 .

The Pacific Journal of Mathematics is issued monthly as of January 1966. Regular subscription rate: $\$ 72.00$ a year (6 Vols., 12 issues). Special rate: $\$ 36.00$ a year to individual members of supporting institutions.

Subscriptions, orders for back numbers, and changes of address should be sent to Pacific Journal of Mathematics, 103 Highland Boulevard, Berkeley, California, 94708.

PUBLISHED BY PACIFIC JOURNAL OF MATHEMATICS, A NON-PROFIT CORPORATION

Printed at Kokusai Bunken Insatsusha (International Academic Printing Co., Ltd.), 270, 3-chome Totsuka-cho, Shinjuku-ku, Tokyo 160, Japan.

Copyright (C) 1973 by Pacific Journal of Mathematics Manufactured and first issued in Japan 


\section{Pacific Journal of Mathematics \\ Vol. 55, No. $2 \quad$ October, 1974}

Walter Allegretto, On the equivalence of two types of oscillation for elliptic

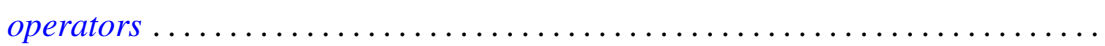

Edward Arthur Bertram, A density theorem on the number of conjugacy classes in

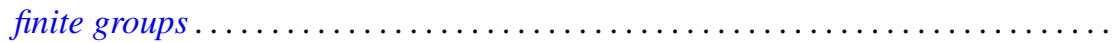

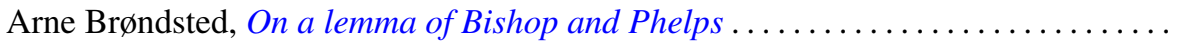

Jacob Burbea, Total positivity and reproducing kernels ..................

Ed Dubinsky, Linear Pincherle sequences . . . . . . . . . . . . . . . . . .

Benny Dan Evans, Cyclic amalgamations of residually finite groups .............

361

Barry J. Gardner and Patrick Noble Stewart, A "going down" theorem for certain

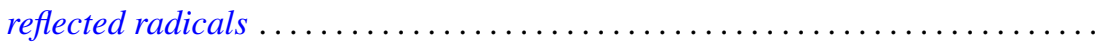

381

Jonathan Light Gross and Thomas William Tucker, Quotients of complete graphs:

revisiting the Heawood map-coloring problem ....................

Sav Roman Harasymiv, Groups of matrices acting on distribution spaces .........

Robert Winship Heath and David John Lutzer, Dugundji extension theorems for

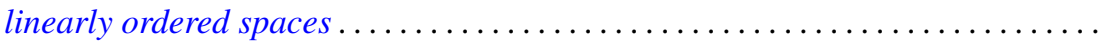

Chung-Wu Ho, Deforming p. l. homeomorphisms on a convex polygonal

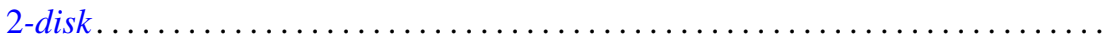

Richard Earl Hodel, Metrizability of topological spaces .................

Wilfried Imrich and Mark E. Watkins, On graphical regular representations of

cyclic extensions of groups .......................... 461

Jozef Krasinkiewicz, Remark on mappings not raising dimension of curves ..... . 479

Melven Robert Krom, Infinite games and special Baire space extensions . . . . . . 483

S. Leela, Stability of measure differential equations . . . . . . . . . . . . . . . . 489

M. H. Lim, Linear transformations on symmetric spaces . . . . . . . . . . . . . . . 499

Teng-Sun Liu, Arnoud C. M. van Rooij and Ju-Kwei Wang, On some group algebra modules related to Wiener's algebra $M_{1} \ldots \ldots \ldots \ldots \ldots \ldots \ldots \ldots \ldots \ldots \ldots$

Dale Wayne Myers, The back-and-forth isomorphism construction ............ 521

Donovan Harold Van Osdol, Extensions of sheaves of commutative algebras by

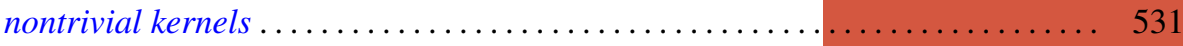

Alan Rahilly, Generalized Hall planes of even order ................... 543

Joylyn Newberry Reed, On completeness and semicompleteness of first countable

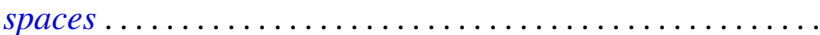

Alan Schwartz, Generalized convolutions and positive definite functions associated

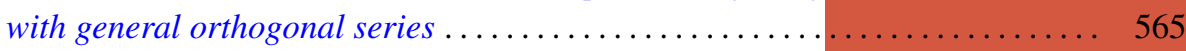

Thomas Jerome Scott, Monotonic permutations of chains . . . . . . . . . . . 583

Eivind Stensholt, An application of Steinberg's construction of twisted groups .... 595

Yasuji Takeuchi, On strongly radicial extensions . . . . ................. 619

William P. Ziemer, Some remarks on harmonic measure in space . . . . . . . . . . 629

John Grant, Corrections to: “Automorphisms definable by formulas” . . . . . . . . 639

Peter Michael Rosenthal, Corrections to: "On an inversion for the general

Mehler-Fock transform pair" ......................... 640

Carl Clifton Faith, Corrections to: "When are proper cyclics injective” . . . . . . 640 